\title{
A RARE CASE OF HEMIPLEGIA
}

\section{General Medicine}

\section{Dr. Valeti}

Rajeswari

\begin{tabular}{ll}
\hline Dr. Kolluru V D & $\begin{array}{l}\text { Postgraduate, Department Of General Medicine, Guntur Medical College Guntur. } \\
\text { *Corresponding Author }\end{array}$ \\
Karthik*
\end{tabular}

\section{Dr. Srinivasula}

Sriranga

\section{Pravallika}

Postgraduate, Department Of General Medicine, Guntur Medical College Guntur

\section{ABSTRACT}

Honey bee sting induced Cerebral infarction is a rare entity. We report a case of 55year old male presented with anaphylactic shock following honey bee sting along with weakness of left side of the body and the face. He was managed with anti histaminics, adrenaline injections, Intravenous fluids, vasopressors, anti platelets and anticoagulants

\section{KEYWORDS}

\section{BACKGROUND}

Stroke is the second most common cause of death and major cause of disability worldwide

Apart from anaphylactic allergic reactions, the sting venom of honeybee can cause hemorrhagic as well as ischemic strokes. These neurological deficits are produced by the contents of venom toxins directly or through the anaphylactic shock or pain induced catecholamines surge.

Regional and systemic reactions cause significant morbidity and mortality.

These reactions have protean manifestations ranging from anaphylaxis, hypotension, rhabdomyolysis, seizures, disseminated intravascular coagulation (DIC) to intracranial haemorrhages and cerebral infarctions, leading to the causation of stroke through different mechanisms.

\section{CASE REPORT}

A 55 year old male patient was found in an unconscious state by the by standers in his paddy fields after which he was brought to ER room.

Vitals status at the time of admission $\mathrm{Bp}-50 / 30$

Pulse rate feeble ( $86 / \mathrm{min})$

Saturation $0289 \%$

Temperature $95.2 \mathrm{f}$

Systemic examination normal except for poor GCS.

After initial resuscitation, his GCS improved to 14/15 ( initially 9/15)

He was treated with vasopressors, IV fluids, Adrenaline, glucocorticoids, antihistamines.

He also had weakness of left hand and left leg associated with slurred speech and confusion
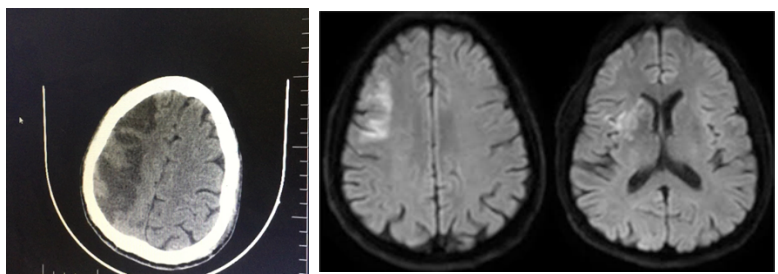

MRI BRAIN with MRA revealed multiple infarcts in arterial territories of anterior and middle cerebral arteries. Small lacunar infarcts in subcortical areas.

MRV found to be normal.
Patient was treated with anti platelets and anticoagulants during hospitalisation.

CBC , FLP, RBS , LFT, KFT , BT CT , PT INR , SERUM ELECTROLYTES are within normal limits.

ECHO was normal.

Carotid artery Doppler was normal

\section{DISCUSSION}

After bee stings, there is an increase in cytokines mainly the interleukins such as IL-1, IL-6, IL-8 and tumor necrosis factor (TNF), which trigger adverse effects on the skeletal muscles, hepatic and renal functions, cardiovascular, central nervous systems.

The bee venom induced neurologic manifestations include stroke, epilepsy, polyradiculopathy, cranial nerve palsies and cavernous sinus thrombosis.

The bee venom also contains thromboxane and leukotrienes, which contribute in vasoconstriction resulting in cerebral infarction.

\section{CONCLUSION}

Bee venom related neurologic events like stroke, are very rare.

Proper neurological examination and immediate correction of hypotension with IV fluids and vasopressors, glucocorticoids can prevent further vascular events.

\section{ACKNOWLEDGEMENTS - Nil}

CONFLICT OF INTEREST- Nil

\section{REFERENCES}

1. Mingomataj EC, Bakiri AH. Episodic haemorrhage during honey bee venom anaphylaxis: Potential mechanisms. J Investig Allergol Clin Immunol. 2012;22(4):237244

2. Moein P, Zand R. Cerebral infarction as a rare complication of wasp sting. J Vas Invent Neurol. 2017; 9(4): 13-16.

Petroianu G, Liu J, Helfrich U, et al. Phospholipase A2-induced coagulation abnormalities after bee sting. Am J Emerg Med. 2000; 18: 22-27.

Yurtseven A, Guvenc Y. Seizure and ischemic attack following bee sting. Turk J Neurol. 2015;21: 138-140. DOI:10.4274/tnd.38991

Andersen KK, Olsen TS, Dehlendorff C, et al. Hemorrhagic and ischemic strokes compared stroke severity, mortality, and risk factors. Stroke. 2009; 40: 2068-2072. DOI:10.1161/STROKEAHA 108.540112 